\title{
La organización sanitaria cubana durante las ocupaciones militares de Estados Unidos
}

\section{Cuban health organization during the US military occupations}

\author{
Carmen Arocha Mariño'; Antonio Castillo Guzmán"'; Benito Pérez Maza'"'
}

\author{
'Doctora en Ciencias de la Salud. Profesora Titular. Escuela Nacional de Salud \\ Pública. La Habana, Cuba. \\ "Máster en Salud Pública. Profesor Auxiliar. Instituto de Cardiología y Cirugía \\ Cardiovascular. La Habana, Cuba. \\ I"Doctor en Ciencias Médicas. Escuela Nacional de Salud Pública. La Habana, Cuba.
}

\section{RESUMEN}

El presente trabajo tiene como objetivo mostrar elementos que permitan comprender la finalidad del gobierno de ocupación militar de los Estados Unidos. En cuanto a las medidas tomadas en relación con la salud pública en Cuba durante las dos ocupaciones realizadas. Se expone la organización que tenía la salud pública en esos momentos y la preocupación del recién instalado gobierno por la situación sanitaria de la Isla, debido a los estragos que le había producido la epidemia de fiebre amarilla a las tropas españolas y a la población cubana, además de los casos de viruela, paludismo, disentería, fiebre tifoidea, tétanos infantil, muermo y otras enfermedades infecciosas. Se concluye que la labor sanitaria realizada durante estas dos etapas está encaminada a garantizar los objetivos económicos y estratégicos de Estados Unidos, íntimamente ligados a la expansión imperialista.

Palabras clave: Salud pública, historia de la salud pública, Cuba. 


\begin{abstract}
This paper was aimed at providing elements to understand the purpose of the US military occupation government in terms of the measures taken in health public care in Cuba during the two US military occupations. The public health organization at that time and the concerns of the government for the health situation in the island due to the ravages of the yellow fever epidemics that had affected the Spanish troops and the Cuban population, in addition to smallpox, malaria, dysentery, typhoid fever, infant tetanus, glanders and other infectious diseases. It was concluded that the healthcare work undertaken during these two phases were directed to protect the US economic and strategic objectives so closely linked to the imperialist expansion.
\end{abstract}

Key words: Public health, public health history, Cuba.

\title{
I NTRODUCCI ÓN
}

No caben dudas de que la sanidad de Cuba tuvo avances significativos durante los dos períodos en que los Estados Unidos (EE.UU.) la ocupó militarmente, a pesar de que en la Primera Ocupación los movía el interés de dominación, y en la Segunda, la necesidad de completar los mecanismos institucionales para lograr la estabilidad.

El Tratado de Paz firmado en París en diciembre del año 1898, fue el inicio de un proceso que convirtió a Cuba en neocolonia de los EE.UU., y condicionó el accionar de los factores sociales internos del país, por lo tanto, al producirse la Primera Intervención y Ocupación Militar al año siguiente, comenzaba a hacerse realidad el sueño del gobierno norteamericano.

La Enmienda Platt, aprobada el 12 de junio de 1901, fue el resultado inmediato de la política de anexión que limitaba la soberanía de Cuba, cuya certidumbre está en las palabras del Gobernador General de la Isla, Leonard Wood cuando expresó: "Cuba debe mantener las condiciones sanitarias que se le han preceptuado, por todo lo cual es bien evidente que está en lo absoluto en nuestras manos."

A continuación se expone un conjunto de elementos que permitirán comprender que la base de la labor del gobierno de ocupación militar, en cuanto a las medidas tomadas en relación con la salud pública, respondía a su interés anexionista.

\section{ORGANI ZACIÓN DE LA SANI DAD}

En 1878 la fiebre amarilla apareció como epidemia de gran severidad en más de cien ciudades de los EE. UU. y se estimaron alrededor de ciento veinte mil ciudadanos enfermos y veinte mil fallecidos, con un monto de alrededor de cien millones de pesos en pérdidas económicas. Toda esta situación dejó el pánico como secuela, causó preocupación en las autoridades y legisladores, como en ninguna 
otra epidemia, ya que existía el criterio erróneo de que esta enfermedad era introducida en la nación procedente de otros países del Sur y Centro América, pero en especial de las Antillas. ${ }^{2}$

La opinión más generalizada entre los médicos norteamericanos, era que la enfermedad la transportaban los barcos que arribaban a sus puertos procedentes de Veracruz, Santo Domingo y Cuba, entre otros. Esta fue la razón de la visita de la Primera Comisión Americana a las Antillas, donde se consideraba que se encontraba el foco originario de fiebre amarilla y desde donde se irradiaba a los EE.UU.

En 1899, al comenzar la Primera Ocupación Norteamericana, en el país había un gran estado de insalubridad y el sistema de salud casi había desaparecido, por lo que se anularon las Juntas de Sanidad que venían del período colonial y se le dio a la salud pública una organización similar a la de los EE.UU., mediante Departamentos de Sanidad creados en el propio mes de enero en La Habana, Santiago de Cuba y algunas otras ciudades.

Los acuerdos de los Departamentos de Sanidad los aprobaban los Ayuntamientos o el Gobernador Militar, pues no había una legislación sanitaria y en 1899 se dictaron varias disposiciones relacionadas con el funcionamiento de la salud pública, entre ellas las órdenes del Gobierno de EE.UU. sobre cuarentenas e inmigración del 17 de enero y 6 de junio respectivamente, el Reglamento General para la Organización de los Servicios Sanitarios Municipales y la Orden Civil No. 15 del 7 de agosto para la creación de la Comisión de Fiebre Amarilla presidida por el doctor Carlos J. Finlay, porque lo reconocían como una autoridad en esta materia, y como secretario, el doctor Diego Tamayo Figueredo (1852-1926), para que confirmara los diagnósticos de casos sospechosos de vómito negro.

Por la preocupación que tenía el recién instalado gobierno, debido a los estragos que le había producido la epidemia de fiebre amarilla a las tropas españolas y a la población en Cuba, además de la presencia de casos de viruela, paludismo, disentería, fiebre tifoidea, tétanos infantil, muermo y otras enfermedades infecciosas, se comenzó la labor de saneamiento en el país. Se nombraron cien médicos cubanos como inspectores para velar por la sanidad de la capital que se dividió a tales efectos en otros tantos distritos. Con la organización que se le había dado se intentaba en esencia controlar las epidemias y proteger las ciudades del sur de los EE.UU., muy cercanas a Cuba. ${ }^{3}$

Debe destacarse que aunque se tomaron muchas medidas, la estructura dada a la salud pública no respondía a la realidad cubana, pues la división por Departamentos impedía la dirección centralizada, lo que obligó a retomar la organización que se tenía del período colonial, de ahí que al siguiente año (1900), se dictara en febrero la Orden Civil No. 13 estableciendo la J unta de Sanidad de La Habana. Dicha Junta no funcionó adecuadamente porque se le mantuvo el poder al Departamento de Sanidad creado al inicio de la ocupación y esto provocó dualidad de funciones, además de que se limitaba de modo general a dirigir los servicios sanitarios municipales, como casas de socorro y asistencia médica a domicilio, entre otros.

Ese año también se produjo la visita de la IV Comisión del Ejército Norteamericano para el estudio de la fiebre amarilla presidida por Walter Reed creada por la Orden especial No. 122, que retomó la teoría de Finlay presentada en la V Conferencia Sanitaria Internacional y trabajó con toda la información del sabio cubano, a partir de lo que pudo emitir sus informes de comprobación en febrero de 1901. 
Ya en el primer semestre de ese año se dictaron las primeras disposiciones de saneamiento con bases científicas dirigidas al exterminio de los mosquitos como agentes trasmisores de la fiebre amarilla, aunque este proceso pudo haber comenzado con anterioridad, pues desde 1881 el doctor Carlos J. Finlay había propuesto la solución a ese problema sanitario. En 1901 además se dictaron medidas de profilaxis de la tuberculosis y se reglamentó la vacunación obligatoria mediante la Orden 165 del 24 de junio, que fue una de las disposiciones sanitarias más importante de esa etapa. ${ }^{4}$

Es de señalar que aunque en el período de la Primera Ocupación Norteamericana se establecieron diferentes servicios y departamentos que beneficiaron a la población, la incipiente unificación lograda con las Juntas de Sanidad en el período colonial se perdió y ni siquiera se interrelacionaron los servicios de la sanidad terrestre con la sanidad marítima, que hasta esos momentos tenían una estructura nacional bien regulada e independiente. No obstante, hubo logros importantes en el cuadro de salud del país, como la eliminación de la viruela y la fiebre amarilla, cuyo último caso autóctono fue diagnosticado el 28 de septiembre de 1901.

Posteriormente, cuando ocuparon el país por segunda vez, el 29 de septiembre de 1906, con la justificación de mediar en la guerra civil que se desató en Cuba, el gobernador Charles Magoon nombró como consejero para el Departamento y la J unta Central de Beneficencia, al comandante Edivin St J ohn Greble y como consultor sanitario, al Coronel J efferson Randolf Kean, con atribuciones en relación con la sanidad como tendría la Secretaría de Gobernación, pues debían tener en sus manos el control sanitario del país. Esta situación provocó nuevamente una duplicidad de funciones, ya que había una estructura sanitaria creada, pero este consultor fue un hombre de amplia visión y presto a escuchar opiniones como las de los doctores Finlay y Barnet, nombrado este último como asesor, quien con el apoyo del Coronel Kean logró que se analizara el fracaso de las juntas locales por la carencia de recursos para el trabajo. ${ }^{5}$

Más tarde, el 26 de agosto de 1907 se promulgó el Decreto No. 894 con el mandato de crear el Departamento Nacional de Sanidad para centralizar las funciones de la Salud Pública Cubana, además de nombrar jefes locales de sanidad y ponerlos bajo la administración del Estado, que desde ese momento quedaba responsabilizado con la situación higiénica de toda la República. En este Decreto, nombrado "Nacionalización de los Servicios Sanitarios", ${ }^{6}$ se expresaba que la conservación de las condiciones sanitarias de la Isla era una obligación nacional y que con la estructura que tenía el sistema de salud en esos momentos, sin uniformidad en los métodos de trabajo, eso no se podía lograr.

A partir de ese momento se organizó el Departamento de Sanidad, y dependiente de el, la Junta Nacional de Sanidad y las Juntas Locales, con J efes Locales de Sanidad en cada municipio, nombrados con todos los recursos, derechos y autoridad para hacer cumplir las Ordenanzas Sanitarias, o sea, se definió la responsabilidad personal dentro de la salud pública y hubo servicios de carácter nacional. Sin lugar a dudas, la organización llevada a cabo fue efectiva porque coincidieron diferentes circunstancias que la favorecieron, pues se logró una buena estructura organizativa, una ley sanitaria bien diseñada que la amparaba y se contó con los medios económicos para desarrollar el trabajo.

Como expone el doctor Jorge Le Roy y Cassá en uno de sus trabajos publicados en 1922, la figura del consultor americano fue de gran importancia para el logro de la "nacionalización de los servicios de salud en Cuba", ${ }^{7}$ aunque a juicio de los autores, en realidad fue el instrumento que facilitó la organización sanitaria nacional, porque la verdadera responsabilidad es de un numeroso grupo de médicos cubanos, que 
comprendieron la necesidad de darle uniformidad a las acciones que se emprendían en el terreno sanitario y no desestimaron ocasiones para gestionarlo.

Otra razón fundamental para que el gobierno de ocupación emprendiera el saneamiento de la Isla con tanto rigor, era la preocupación que tenía la burguesía comercial y financiera norteamericana en relación con el puerto de La Habana, por ser un punto estratégico para el comercio del Caribe; la presencia de las enfermedades antes mencionadas se convertía en una barrera.

Resulta muy importante para demostrar cual era el interés que tenía el Gobierno de EE.UU. por organizar los servicios de salud, un informe emitido al finalizar la primera ocupación norteamericana, por William C. Gorgas, Jefe de la Sanidad del Ejército de ese país en Cuba, al Brigadier General Leonard Word, donde señalaba que:

...el objeto principal del saneamiento, en cuanto interesaba a los Estados Unidos, era la extirpación de esta enfermedad, que por más de doscientos años había causado grandes pérdidas de vidas y la completa paralización del comercio durante las epidemias. ${ }^{8}$

En dicho informe el Comandante Gorgas apuntaba también que:

...en 1900 la ciudad se encontraba perfectamente limpia y en las mejores condiciones sanitarias posibles y a principios de 1901, el Departamento de Sanidad se convenció que las medidas sanitarias ordinarias no afectaban en nada a la fiebre amarilla, además, en 1898, primer año de estar instalados en Cuba, con un promedio de 8343 hombres, la mortalidad por enfermedades ascendió a 67,94 por mil.

Es decir, que con las medidas tomadas la mortalidad general descendió, pero los casos de fiebre amarilla se mantenían igual, pues aún se desconocía la causa que la producía, y los "yanquis" acusaban a Cuba de ser uno de los países más insalubres de la tierra, pero en realidad lo era tanto como las propias regiones del Centro y Sur de los EE.UU.

Otra cuestión que motivaba el interés del gobierno norteamericano por garantizar la salubridad de Cuba, es que en esos momentos la I sla llegó a ocupar el primer lugar entre los países latinoamericanos receptores de su capital, situación que demandaba condiciones higiénicas apropiadas para el proceso inversionista, cuyo monto alcanzó las siguientes cifras: ${ }^{9}$

Inversiones norteamericanas en Cuba hasta 1906

\begin{tabular}{|l|c|}
\hline Bajo gobierno español: & $\$ 50$ millones \\
\hline Gobierno ocupación: & $\$ 30$ millones \\
\hline Primera República: & $\$ 80$ millones \\
\hline
\end{tabular}

Distribución de las inversiones en 1906 


\begin{tabular}{|l|c|}
\hline Industria azucarera: & 30 millones \\
\hline Industria tabacalera: & 30 millones \\
\hline Industria ganadera: & 30 millones \\
\hline Industria ferrocarriles: & 39 millones \\
\hline Bienes raíces y tierras incultas: & 11,5 millones \\
\hline Otros: & 60 millones \\
\hline Total: & 200 millones \\
\hline
\end{tabular}

Debido a todo este proceso las relaciones mercantiles también se incrementaron y muchos ciudadanos del norte se establecieron en Cuba, además de las frecuentes visitas de otra parte de su población a la que había que proteger, al mismo tiempo que a las tropas de ocupación y a la fuerza de trabajo que les era necesaria para garantizar sus intereses. Sólo en 1905 se estimaba que 13000 colonos norteamericanos habían adquirido tierras en la Isla. ${ }^{10}$

De no menos importancia para el gobierno de los EE.UU. en el terreno político, era que esta labor de saneamiento le permitía justificar su permanencia en Cuba, a la vez que le servía para mostrarle al mundo las ventajas que reportaban las medidas que en materia de salud pública se tomaban en los países ocupados por sus ejércitos.

Finalmente se puede concluir que el proceso organizativo que caracterizó a los servicios de salud en Cuba durante las dos ocupaciones norteamericanas, estuvo dirigido a crear las condiciones para llevar a cabo acciones efectivas de saneamiento y de esta manera librar a la Isla de epidemias que fueran obstáculos para garantizar los objetivos económicos y estratégicos de EE.UU., íntimamente ligados a la expansión imperialista.

\section{REFERENCI AS BI BLI OGRÁFI CAS}

1. Le Riverend J. Historia Económica de Cuba. La Habana: Editorial Pueblo y Educación; 1975.

2. López Sánchez J. Finlay. El hombre y la verdad científica. La Habana: Editorial Científico Técnica; 1982.

3. Delgado García G. Conferencias de Historia de la Administración de Salud. Cuad Hist Salud Pública. No. 81. 1996.

4. López del Valle JA. La Nacionalización de los Servicios de Salud. La Habana: Oficina del Historiador del MINSAP; 1908. [Discurso leído en la sesión solemne celebrada por la Junta de Sanidad para descubrir los retratos de los señores Charles E. Magoon, JR. Kean y Carlos J. Finlay, el día 7 de marzo de 1908].

5. Delgado García G. Conferencias de Historia de la Administración de Salud. Cuad His Salud Pública. No. 81. 1996. 
6. Gaceta Oficial de la República de Cuba, bajo la Administración Provisional de los Estados Unidos. Agosto 29 de 1907. Tomo II: 2049-54.

7. Le-Roy y Cassá J. Desenvolvimiento de la Sanidad en Cuba durante los últimos cincuenta años. (1871-1920). La Habana: Imp. 'La moderna Poesía"; 1922.

8. Informe Civil del Brigadier General Leonard Wood. Gobernador Militar de Cuba. Enero 1ro. a Mayo 20 de 1902. Tomo I. La Habana: Oficina del Historiador del MI NSAP.

9. Pichardo Viñals H. Documentos para la Historia de Cuba. La Habana: Editorial Ciencias Sociales; 1976.

10. Le Riverend J. Historia Económica de Cuba. La Habana: Editorial Pueblo y Educación; 1975.

Recibido: 2 de septiembre de 2008.

Aprobado: 6 de noviembre de 2008.

Carmen Arocha Mariño. Escuela Nacional de Salud Pública. Calle Línea esq. I. El Vedado. La Habana 10400, Cuba.

E-mail: carmen.arocha@infomed.sld.cu 